\title{
DISEÑO ESTRUCTURAL PARAMETRICO
}

\section{PARAMETRIC STRUCTURAL DESIGN}

\author{
Ing. Farez, Jorge ${ }^{2}$, Arq. Lordella, Patricio ${ }^{3}$, Arq. Fostel, Juan ${ }^{4}$. \\ Colaboradores \\ Ing. Langer Patricia, Arq. Chichizola Aldana, DelMoro Lucia. \\ Facultad de Arquitectura y Urbanismo - UNLP, La Plata, Argentina. \\ jorgeefarez@gmail.com, arqui_pato@live.com.ar, juanfostel@gmail.com
}

\section{RESUMEN}

En el presente documento se describe la creación de una metodología de diseño arquitectónicoestructural utilizando el modelado paramétrico, combinado con un análisis del comportamiento de los sistemas resistentes que se evalúan mediante optimizadores multi-objetivo. Esto aporta una herramienta para la exploración de un amplio espacio de diseño, como proceso de toma de decisiones y como aporte para la comprensión del funcionamiento estructural.

Los resultados obtenidos muestran el intercambio de información en tiempo real entre los parámetros de la geometría creada por la arquitectura y evaluada con métodos de la ingeniería, utilizándose principalmente en el área de la educación universitaria y orientada a la enseñanza conceptual de las estructuras.

El modelado arquitectónico se realiza teniendo simultáneamente los datos de la estructura, aportándole de esta manera herramientas al diseñador, que permiten ampliar las fronteras creativas para la materialidad del proyecto.

\section{ABSTRACT}

This document describes the creation of an architectural-structural design methodology using parametric modeling, combined with an analysis of the behavior of resistant systems that are evaluated using multi-objective optimizers. This provides a tool for exploring a wide design space, as a decisionmaking process and as a contribution to the understanding of structural functioning.

The results obtained show the exchange of information in real time between the parameters of the geometry created by the architecture and evaluated with engineering methods, being used mainly in the area of university education and oriented to the conceptual teaching of structures.

The architectural modeling is carried out simultaneously having the structure data, thus providing tools to the designer, which allow expanding the creative boundaries for the materiality of the project.

\footnotetext{
${ }^{2}$ Profesor Titular Cátedra Estructuras FLL. Facultad de Arquitectura y Urbanismo, Universidad Nacional de La Plata

${ }^{3}$ Docente de Curso Diplomado Cátedra Estructuras FLL - Facultad de Arquitectura y Urbanismo, Universidad Nacional de La Plata

${ }^{4}$ Docente de Curso Diplomado Cátedra Estructuras FLL - Facultad de Arquitectura y Urbanismo, Universidad Nacional de La Plata
} 
PALABRAS CLAVES: parametricismo - estructuras - optimizadores - sustentabilidad.

KEY WORDS: parametricism - structures - optimizers - sustainability.

Artículo RECIBIDO: 21/07/19 | Artículo ACEPTADO: 15/11/19

\section{INTRODUCCIÓN}

El arquitecto es el responsable de crear un diseño de alto rendimiento siendo éste el resultado de múltiples objetivos Venustas (belleza), Firmitas (firmeza) y Utilitas (utilidad) como proponía en su tratado "De Architectura" Marco Vitruvio.

A menudo éstos objetivos compiten entre sí y deben ser todos cuidadosamente equilibrados. Los avances en el diseño digital han traído una nueva libertad de diseño en la arquitectura, del mismo modo, la proliferación de herramientas de software de análisis ha ayudado a los ingenieros a calcular modelos estructurales complejos, permitiéndoles no solo realizar un análisis más exacto de su funcionamiento, sino también acelerando el proceso de análisis obteniendo resultados de tensiones, momentos y deformaciones en tiempo real con cálculos que utilizan elementos finitos.

Nuestra propuesta consiste en incorporar desde el diseño arquitectónico preliminar los aspectos estructurales con herramientas paramétricas sencillas que dan resultados rápidos y certeros.

De esta manera los cálculos de ingeniería que participan en el análisis y la evaluación se integran con los parámetros de diseño arquitectónico desarrollando, desde las primeras etapas del proyecto, las pautas para incorporar los conceptos y necesidades estructurales en las decisiones proyectuales. Se complementan las evaluaciones utilizando optimizadores que tienen en cuenta los distintos objetivos que condicionan al diseño arquitectónico y estructural, poniendo especial énfasis en los aspectos de sustentabilidad en cuanto al uso de materiales estructurales. Se utilizan software como el Octopus y el Galápagos, que son de uso libre en la web y en especial el Zirkel, desarrollado por nuestro estudio para ser utilizado como herramienta multi-objetivo de evaluación con diferentes metas de diseño, construcción y eficiencia sustentable.

\section{DESARROLLO}

\subsection{Objetivos}

Desarrollo de una herramienta de trabajo y producción con el propósito de ser utilizada en la etapa de proyecto de la obra arquitectónica que permita optimizar el proceso de diseño estructural para lograr un mejor aprovechamiento, entre otros aspectos, de la energía consumida antes, durante y finalizada la vida útil de la obra en estudio, con aplicación en el ámbito profesional y en el académico.

\subsection{Equipo de trabajo}

Nuestro equipo está conformado por arquitectos, ingenieros y programadores, que realizan un trabajo multidisciplinar de investigación y docencia, incorporando experiencias en la realización de proyectos y construcciones civiles.

\subsection{Método}


En el presente escrito se plantea, además, la utilización de optimizadores que se desarrollaron en nuestro equipo de trabajo para ser utilizados como un colaborador digital que nos orienta rápidamente sobre las mejores posibilidades de la estructura ideada para el proyecto en ejecución. Zirkel, tiene el objetivo de orientar al proyectista sobre cuáles son las mejores poblaciones de opciones dentro los objetivos particulares impuestos.

En el esquema estructural de la obra en la Figura 1, se plantea la utilización del optimizador estructural Zirkel (Brújula de Búsqueda), que nos permite verificar y analizar todas las variables teniendo en cuenta los aspectos estructurales, funcionales y constructivos impuestos como prioridad por el proyectista en cada objetivo.

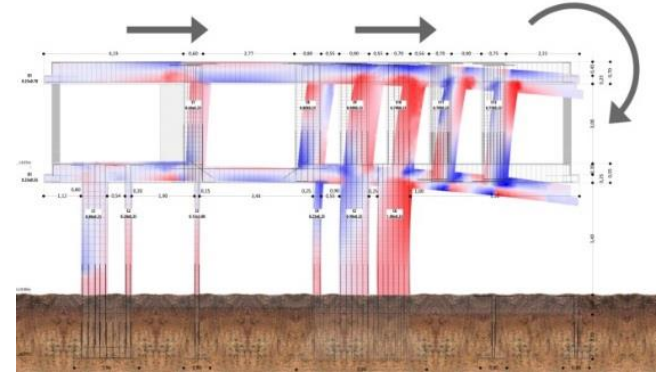

Figura 1. Obra en estudio. Modelado estructural

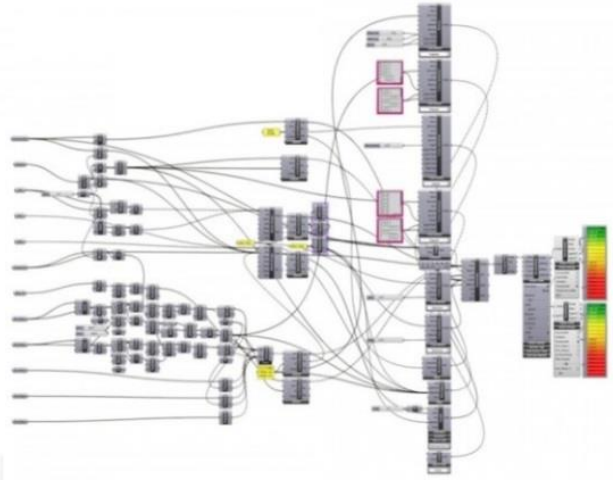

Figura 2. Desarrollo en Grasshopper

El termino parametricismo implica que todos los elementos del modelo son modificables, tanto en su posición como en su dimensión, orientación, forma, color, etc.; y que a la vez todos éstos elementos están interrelacionados.

El Diseño Estructural Paramétrico utiliza este modelo de herramienta proyectual y los conecta a un visualizador, a un analizador estructural y a un optimizador, permitiendo modificar las variables definidas en el proyecto, contando al mismo tiempo con los resultados que surgen de las modificaciones de los parámetros del proyecto. De esta manera se obtiene, en forma instantánea, los resultados estructurales de las modificaciones que se realizan con el ajuste del proyecto permitiendo un dinamismo y una búsqueda más libre y creativa a la vez. (Figura 2 y Figura 3)

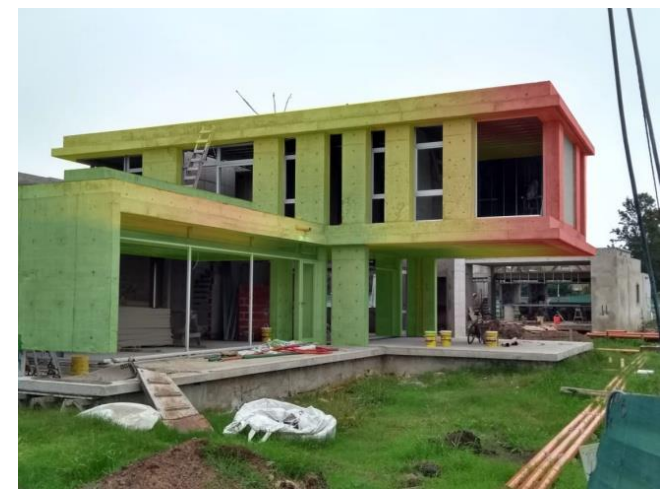

Figura 3. Obra terminada. Los colores identifican las deformaciones

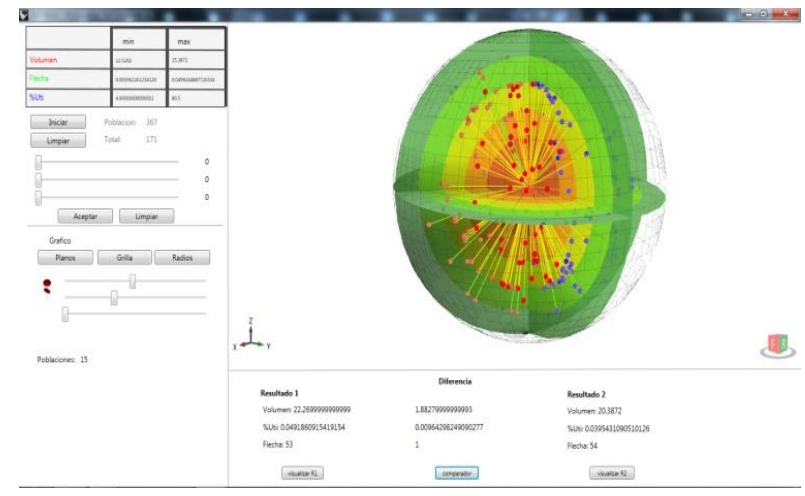

Figura 4. Brújula de búsqueda "Zirkel" con las poblaciones de posibles soluciones estructurales Este análisis en forma simultánea permite contar de manera inmediata con los datos necesarios para evaluar la viabilidad de la idea arquitectónica. Por esta razón, el proyecto en lugar de ser una forma 
terminada, es el resultado de una determinada cantidad de parámetros y funciones programadas que pueden modificar instantáneamente sus valores dentro del rango impuesto a cada parámetro. La Herramienta Paramétrica permite desarrollar nuevos conceptos, tanto en los aspectos proyectuales, de diseño estructural como de Enseñanza de las estructuras. Esta última rama es donde más nos hemos focalizado, teniendo en cuenta las necesidades de los estudiantes y profesionales de la construcción se ha orientado el uso de la Herramienta Paramétrica, además, hacia la modelación desde visualizadores, como el Rhino, permitiendo al estudiante y al arquitecto tener, en tiempo real y a medida que va esbozando su proyecto, los valores tensionales y de deformaciones de la estructura que se está creando.

\subsection{Sustentabilidad.}

La utilización de la herramienta paramétrica como elemento de toma de decisiones, con sus Plugins desarrollados a propósito de las necesidades proyectuales, no debe acotar ni generalizar o poner un sello común a los proyectos; debe permitir la posibilidad de explorar nuevos horizontes que plantea el dinamismo de los paradigmas actuales.

Las condicionantes en las que la Arquitectura se desarrolla cambian permanentemente, generando una necesidad de dinamismo en los recursos proyectuales que hace imprescindible la incorporación de nuevas tecnologías que respondan adecuadamente a los nuevos paradigmas. La estructura; debe dar respuesta e incorporarse a la necesidad de sustentabilidad, evaluando y analizando los sistemas estructurales tradicionales, incorporando como elemento de análisis, no solamente nuevas materialidades sino elementos comparativos de consumo energético de fabricación y su huella ecológica.

La incorporación de optimizadores y comparadores digitales para medir la eficiencia, no solo deben tener en cuenta el consumo volumétrico y pesos estructurales, sino también su gasto energético de fabricación y su efecto en el medio ambiente formando parte de una exigencia para la evaluación de la eficiencia de los proyectos.

Los plugins desarrollados van en búsqueda, no solamente de convertirse en una herramienta eficaz de uso para optimizar los proyectos y maximizar la formación en los aspectos estructurales a los futuros Arquitectos, sino que deberían ser también utilizados para medir a los proyectos en su eficacia sustentable.

Esta línea de investigación surge de preguntarnos, ¿cómo podemos crear edificios más eficientes? Si bien es un tema muy investigado en estos tiempos, generalmente para el análisis de sustentabilidad que se hace para una edificación se tiene en cuenta su vida útil y el consumo energético de su uso en ese lapso. Nuestra propuesta consiste en encontrar soluciones previas al uso del edificio, por lo que proponemos analizar la optimización desde el proceso de diseño, utilizando el optimizador multi objetivo Zirkel (Figura 4).

La eficacia estructural de distintos materiales en terminos de la energia que se necesita para fabricarlos. (Tabla 1). 
Ing. Farez, Jorge ${ }^{1}$, Arq. Lordella, Patricio ${ }^{1}$, Arq. Fostel, Juan ${ }^{1}$

Material Energia necesaria para asegurar Una determinada rigidez al conjunto de la estructura

Acero

Aluminio Ladrillo

Hormigon Madera
1

4

0.4

0.3

0.02
Energia necesaria para producir un panel de una tension de rotura a Compresion deter. 1

2

0.1

EWDS $\quad 0.05$

.Fuente: J.E.Gordon Estructuras

\section{Impacto de la construcción}

Como bien sabemos todos los sectores económicos de la sociedad tienen una influencia, generalmente negativa, sobre el medio ambiente.

El sector de la construcción como veremos en el siguiente gráfico (Figura 5), es uno de los que mayormente repercute en los niveles de contaminación ambiental, por este motivo hace un tiempo se viene promulgando la sustentabilidad edilicia.

\section{Impacto de la construcción mundial}

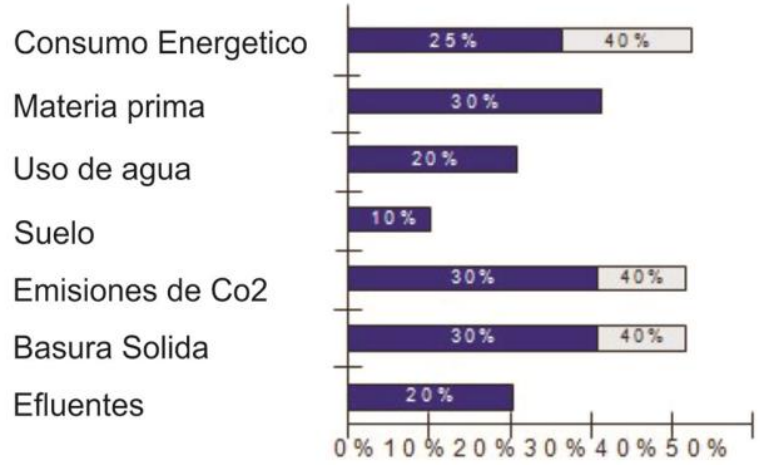

Figura 5. Ref. Datos de las Naciones Unidas

Los materiales de la construcción inciden en el medio ambiente a lo largo de su ciclo de vida, desde su primera fase (extracción) hasta el final de su vida útil (tratamiento como residuo), pasando obviamente por todas sus fases intermedias que veremos a continuación.

Si se suman todas sus etapas, la construcción es el sector con mayor Huella Ecológica de nuestro plantea. Conforme a los datos de las naciones unidas, la construcción contribuye hasta en un $30 \%$ en las emisiones anuales de gases de efecto invernadero, consume hasta un $40 \%$ de la energía, extrae un tercio de los materiales del medio natural, genera el $40 \%$ de los residuos sólidos urbanos, consume un $12 \%$ del agua y ocupa el $12 \%$ del territorio del planeta. Además, económicamente es responsable del 10\% del PIB mundial (BENITE, 2011).

\subsection{Optimizadores.}

Todo diseñador piensa de manera paramétrica, busca la mejor relación entre parámetros priorizando 


\section{DISEÑO ESTRUCTURAL PARAMETRICO}

Ing. Farez, Jorge ${ }^{1}$, Arq. Lordella, Patricio ${ }^{1}$, Arq. Fostel, Juan

los que cree necesarios, dándole como resultado unos pocos modelos. El sistema paramétrico busca resolver el proceso de pensamiento permitiendo modificar de manera rápida los parámetros y prioridades, dando automáticamente la mayor cantidad de resultados posibles.

Grasshopper es un editor gráfico algorítmico relacionado con herramientas de modelado 3D de Rhinoceros, este programa de código abierto permite no solo a los diseñadores crear y programar componentes, sino también realizar un trabajo multidisciplinar donde ingenieros en sistemas configuran funciones específicas para facilitar el análisis y la elaboración de tareas.

En el inicio del proyecto las técnicas paramétricas permiten análisis de comportamiento estructural energético en forma general. Esta aproximación se conoce como diseño generativo, ya que a través de distintos procesos algorítmicos se genera una forma según criterios de evaluación establecidos. Los resultados obtenidos deberán ser validados por análisis tradicionales que permitan verificar las normativas vigentes y también revisar su ejecución. (Figura 6)

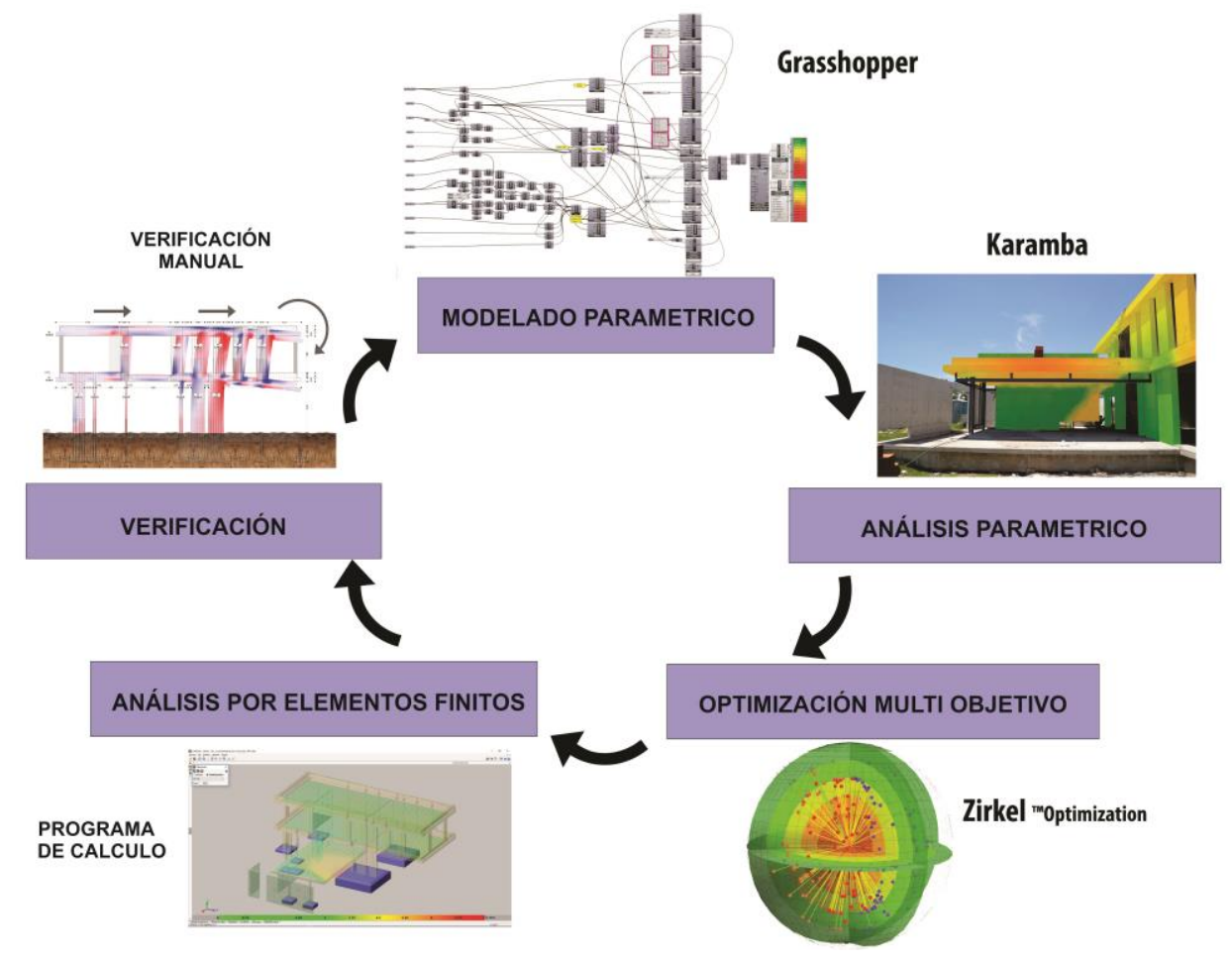

Figura 6.

\section{6 enseñanza.}

Esta nueva forma de enseñar está siendo aplicada desde hace más de cinco años en la asignatura Estructuras III y en el Curso de Posgrado de Diseño Estructural Paramétrico dictado en la Facultad de Arquitectura y Urbanismo de la Universidad Nacional de La Plata (FAU UNLP), Buenos Aires, Argentina. (Figura 7; Figura 8; Figura 9, Figura 10 y Figura 11). 


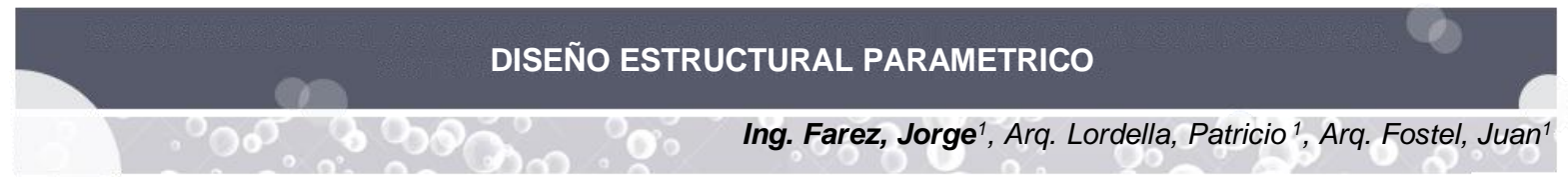

Estructuras parametrizadas para la conceptualización estructural

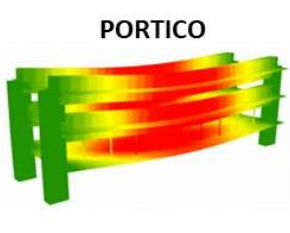

ARCO Y FUNICULAR
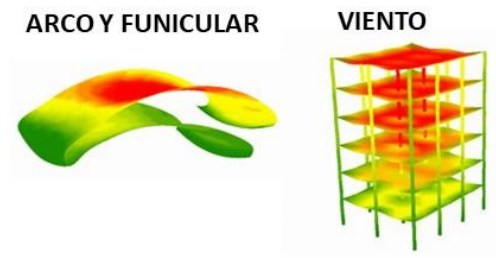

VIEREDEEL

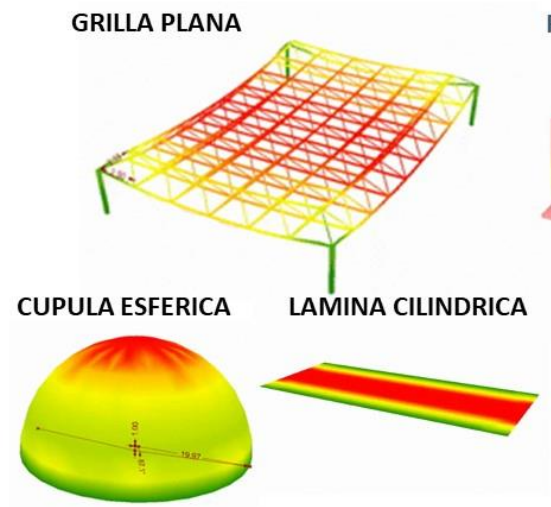

FUNDACIONES
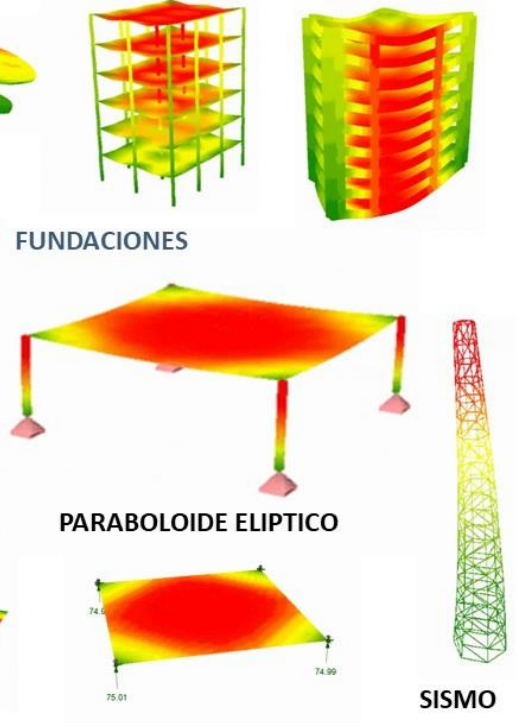

Figura 7.

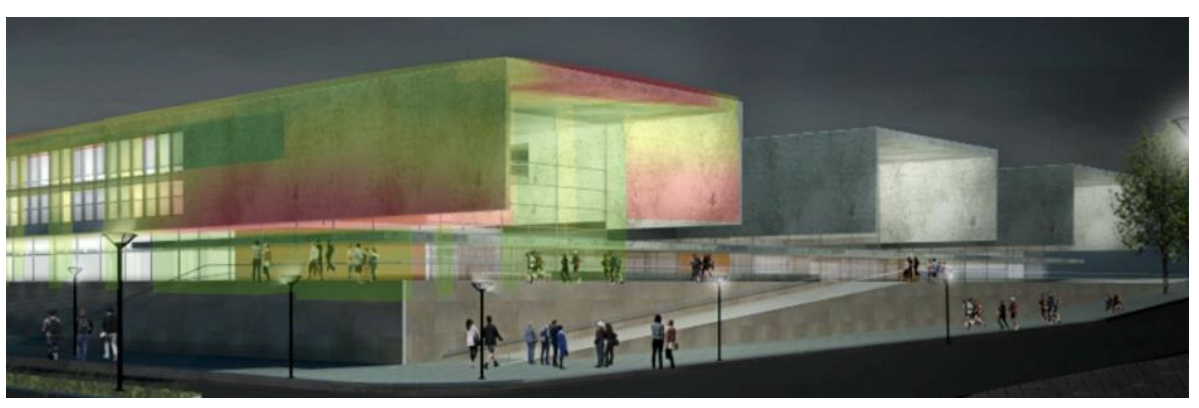

Figura 8. Concurso "Complejo Tribunal en Necochea" 3er. Premio Arqs. Santinelli y Squillacioti

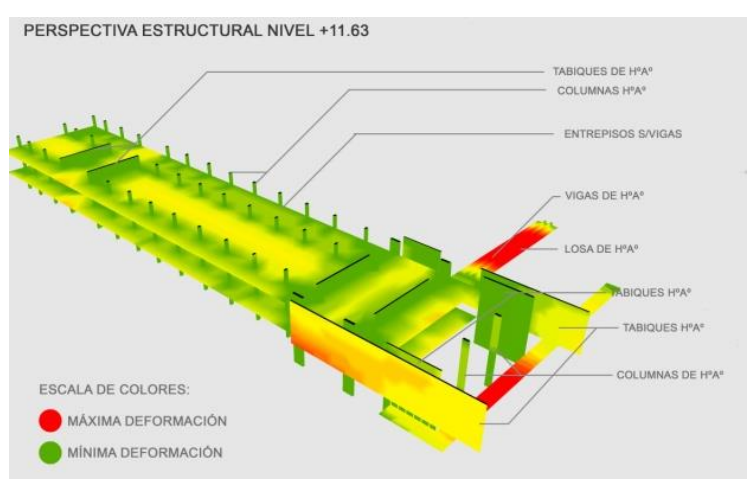

Figura 9. Concurso "Complejo Tribunal en Necochea"

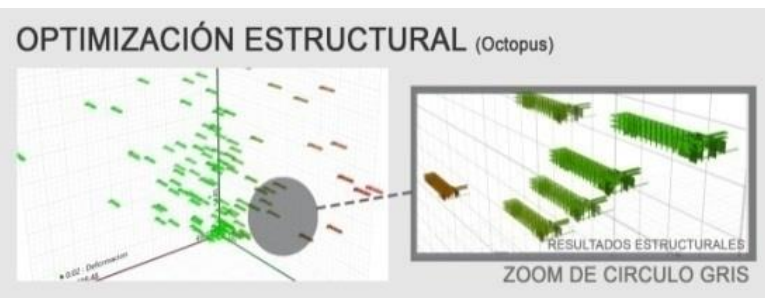

Figura 10. Proceso de optimización

Diseño Estructural Paramétrico: Ing. J.Farez - Arq. P. Lordella. 


\section{DISEÑO ESTRUCTURAL PARAMETRICO}

Ing. Farez, Jorge ${ }^{1}$, Arq. Lordella, Patricio ${ }^{1}$, Arq. Fostel, Juan

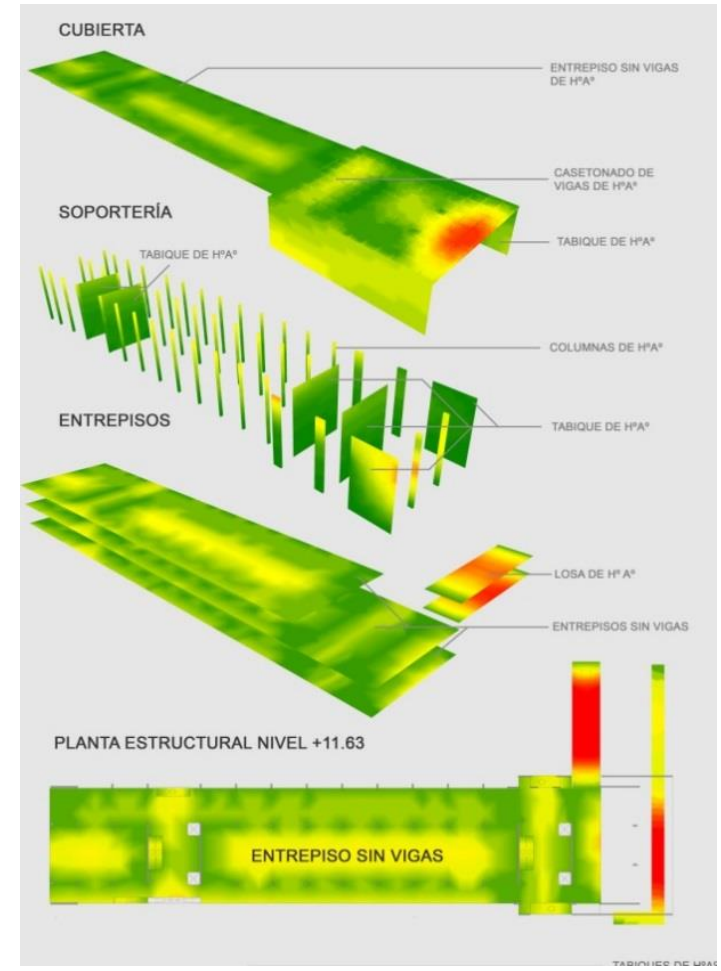

Figura 11. Concurso "Complejo Tribunal en Necochea" Diseño Estructural Paramétrico: Ing. J.Farez - Arq. P.Lordella

\subsection{Zirkel:}

Herramienta de uso conceptual para la compresión del funcionamiento estructural desarrollado y orientado a evaluación de proyectos estructurales y enseñanza de las Estructuras para estudiantes de Arquitectura. (Figura 12).

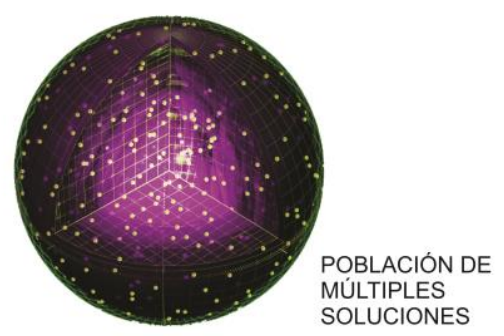

Figura 12. Zirkel $\circledast$ optimizador estructural multi - objetivo desarrollado por nuestro equipo

Reconocimiento:

- Facultad de Arquitectura y Urbanismo UNLP, Buenos Aires, Argentina

- Preisinger-Clemens consultores para problemas con Karamba.

- David Rutten consultor para componentes de Grasshopper. 


\section{Conclusiones}

Los resultados de esta investigación describen metodologías de trabajo y métodos para evaluar las propuestas alternativas de diseño en etapas tempranas del proyecto arquitectónico, obteniendo en tiempo real datos imprescindibles para la libertad proyectual y la toma de decisiones. Una forma arquitectónica no puede ser analizada solo en base a su comportamiento estructural, debería ser evaluada a través de procesos de simulaciones estructurales que contemplen los costos energéticos, contaminación ambiental, tiempos de producción etc.

El uso de Zirkel y el comparador nos da un acercamiento y comprensión conceptual del funcionamiento estructural permitiendo obtener las poblaciones de resultados que sean más adecuadas a las expectativas propuestas por el proyectista.

\section{Bibliografía}

Moussavi, Farshid (2016). The Function of Form. EE.UU. Ed. Actar and the Harvard University Graduate School of Design

Gordon, J.E. (1999). Estructuras O Por Que Las Cosas No Se Caen. Ed. Celeste Ediciones Wagensberg, Jorge (2013). La rebelión de las formas. Ed. Tusquets Editores S.A.

Schamun, Jorge M. (1999) Introducción al método de los elementos finitos Edit. CEILP-UNLP

\section{Referencias / links de interés}

- https://www.karamba3d.com/ David Rutten

- http://estructurasfll.wixsite.com/unlp

- http://www.grasshopper3d.com/

- http://www.innovativeresearchpublication.com/documents/papers/Dubai2016/ICSACE paper 5.pdf Proceedings of 2nd International Conference on Structural Architectural and Civil Engineering, Held on 19th - 20th November 2016, in Dubai, U.A.E ISBN: 9788193137376

- Congreso Regional de Tecnología en Arquitectura CRETA X (29, 30 y 31 de agosto de 2018) Facultad de Arquitectura y Urbanismo - Universidad Nacional de La Plata, Provincia de Buenos Aires, República Argentina.

- Congreso Regional de Tecnología en Arquitectura CRETA XI "Tecnologías para una Arquitectura Regionalmente Sustentable" (28, 29 y 30 de agosto de 2019) Facultad de Arquitectura, Urbanismo y Diseño - Universidad Nacional de Mar del Plata, Provincia de Buenos Aires, República Argentina 